\title{
Structural and Characteristics Change of Water Hyacinth Fiber due to Combine Effect of Plasma and Nano-technology
}

\author{
Hari Prakash. N, Mariammal. M, Infant Solomon, Bornali Sarma, Arun Sarma* \\ Division of Physics, School of Advanced Sciences (SAS), VIT University Chennai campus, Chennai 127, Tamilnadu, India \\ *Corresponding author: arunkumar.sarma@vit.ac.in
}

\begin{abstract}
Water hyacinth [Eichhornia crassipes] fiber has been treated using DC glow discharge plasma to make its surface more hydrophobic in nature for various applications. The plasma treated fibers have been coated with synthesized phase pure $\mathrm{ZnO}$ nano-particle at room temperature to enhance both hydrophobicity and Ultra Violet [UV] light protection rate. The behavioral changes of fibers and its characteristics have been studied using XRD, SEM, EDS, a ATR-FTIR and TGA. Surface chemistry of the treated fiber has been altered as depicted by ATRFTIR studies, while the improve degree of crystallinity analysed by XRD shows the effects of molecular structure of the fibers. The hydrophobic nature of the fibers has been recognized by contact angle measurement using Goniometer and by water absorption processes. Moreover, moisture content of treated fiber has been measured quantitatively using TGA. Finally wavelet analysis has been applied to understand the surface characteristics of water hyacinth fiber and compare its surface roughness value was measured by SEM. Furthermore, entropy of the treated and untreated fibers has also been calculated.
\end{abstract}

Keywords: contact angle, hydrophobicity, moisture, plasma treatment, wavelet analysis

Cite This Article: Hari Prakash. N, Mariammal. M, Infant Solomon, Bornali Sarma, and Arun Sarma, "Structural and Characteristics Change of Water Hyacinth Fiber due to Combine Effect of Plasma and Nano-technology." American Journal of Materials Science and Engineering, vol. 5, no. 1 (2017): 17-27. doi: 10.12691/ajmse-5-1-3.

\section{Introduction}

To avoid global warming on environment, natural fiber such as water hyacinth was replaced by some nonbiodegradable and renewable fibers [1]. Water hyacinth, scientifically known as Eichornia Crassipes was first observed in the part of meridia [2] and is a free drifting amphibian and obtrusive wild plant [3] are now almost everywhere throughout the world. Water hyacinth becomes quick and edges of its tangle can even increments by 60 centimeter per month in favourable conditions [4]. It has an important advantage of being a sustainable resource for bio-fuel production because of lingo-cellulosic structure. However, not much work has done with such fiber for proper applications till date. There are various available technological options for renewable fuel from water hyacinth $[5,6,7]$ such as hydrolysis [8,9] fermentation [10,11] HTL and HTG $[12,13]$ etc. On the other hand, water hyacinth fiber can also be used for high moisture biomass feedstock to have liquid fuel [14]. More importantly, it can be use to replace the petroleum based fibers which are the cause of global warming.

The main idea of this study is to treat the water hyacinth fiber in plasma environment to enhance its water absorption capacity and hence its hydrophobicity using both plasma and thin film nano-technology simultaneously. To highlight the environment related issues it is important to use plasma and nanotechnology together to get almost pollution free environment [15]. Natural fibers, like water hyacinth has attracted more attention in recent years for wide acceptable applications in science and engineering.

Glow discharge plasma has the efficient creator of free radicals and having enough energy to change the bonds of any fibers and more specifically on polymer based fibers. Plasma technology is being extensively used to textile and polymers [16,17]. Again, inorganic materials, for example, metal and metal oxides are being considered for many applications on fibers because of their capacity to resist actions [18]. Some materials/metal oxides like $\mathrm{TiO}_{2}, \mathrm{ZnO}, \mathrm{MgO}$ and $\mathrm{CaO}$ are behaving steady under hostile process conditions and also considered as protected materials [19]. For instance, Ag nano-particles are used for stop irresistible sicknesses because of its antimicrobial properties. Many of these properties of metal nano-molecule are depending on size, crystallinity, morphology of particles, shape and creation [20].

To understasnd surface morphology more clearly, wavelet transform (WT) has been adopted through signal and image processing. Wavelet transformation can help us to meet information on surface morphology and topography [21], which is considered in localized space and frequency. Using the algorithm multi-resolution signal decomposition (MRSD), WT can extend the image 
information directly at different resolution for enabling interpretation of the image details from a lower-to-higher resolution [22].

This work has been done to undersand the structural changes of water hyacinth fibre by introducing plasma and nano-technologies together. Various characterisation such as, XRD, SEM, EDS, FTIR-ATR and TGA have been done to undertand the phenomena. The altered surface chemistry of the treated fibers were observed that FTIRATR. The degree of crystallinity has also improved which was been analysed by XRD and shows the effects of super molecular structure of the fibers. In addition, the hydrophobic nature of the fibers has been recognized by contact angle measurement using Goniometer and by water absorption processes. Moreover, moisture content of treated fiber has also been deduced quantitatively measured by TGA. Wavelet analysis has been adopted to understand the surface characteristics of water hyacinth fiber and its corresponding entropy value has been calculated for both treated and untreated fibers.

\section{Theory}

\section{Wavelet Analysis (WT)}

Surface roughness of both space and frequency areas can be clearly understood using wavelet analysis. WT analysis can provide multi-scale morphological picture related to any frequency data $[21,22,23]$. It normally provides verifiable capacity of non-stationary signals [24]. Any discrete form of wavelet is expressed as

$$
\psi_{j, k} \frac{1}{\sqrt{s_{0}^{j}}}=\psi\left(s_{0}^{-j} x-k \gamma_{0}\right)
$$

Where $\mathrm{S}=s_{0}^{j}$ and $\gamma_{0}$ a the dilation scale parameter is the translation parameter where the localization parameters are represented by $j$ and $k$ respectively. Any energy limited signal $f(x)$, the corresponding the discrete wavelet transform (DWT) is expressed in Eq. (2) and the respective signal processing is performed by using DWT as

$$
c_{f}(j, k)=\int_{-\infty}^{+\infty} f(x) \psi(x) d x .
$$

\section{Multiresolution signal decomposition (MRSD)}

Grossman and Morlet [25] have developed WT using different algorithms. They proposed the CWT, based on translation and dilation, where signals are decomposed due to both the space and the scale domains. Later on, Mallat [22] introduced the algorithm of multi resolution signal decomposition (MRSD), which is mostly used for all signal processing. Original signals were decomposed in MRSD by connecting orthogonal wavelets. In this process, signals are investigated at various frequency groups with various resolutions by decomposing to low and high frequency component $\left(A_{n}^{l}, D^{l}{ }_{n}\right)$ and repeated until the craved scale level of signal segment is accomplished. These low-and high-frequency segments at any position can be expressed as,

$$
\begin{aligned}
& A_{n}^{k}=\sum_{j=n-N}^{n} A_{j}^{k-1} h_{j-n} \\
& D_{n}^{k}=\sum_{j=n-N}^{n} A_{j}^{k-1} g_{j-n}
\end{aligned}
$$

Where $n$ is the sampling position, $k$ is the scale level, $k=1$, 2, 3 . . and $N$ is the sampling number. For this calculations, Daubechies wavelet of order 8 (MATLAB) has been used as the basic function to analyze the signal.

Information gethered from the available output decomposed signals, parameters like entropy or energy can also be calculated using formula

$$
E_{k}^{i}=\sum_{i=1}^{n}\left[P_{k}^{i}(x)\right]^{2}
$$

where $i$ is the packet number and $k$ is the level number of the decomposed wavelet packet required contain some higher energy. The decomposed wave packets surface roughness has been calculated and compared with already measured roughness value. In this study, DWT has been executed by applying a process of MRSD of the signal [22]. Surface roughness of processed fibers can be efficiently calculated using this process.

\section{Experimental Setup}

\section{Synthesis of Zinc Oxide}

$\mathrm{ZnO}$ nano particle were prepared through chemical synthesis of Zinc chloride $(\mathrm{ZnCl})$ after neutralizing with sodium hydroxide $(\mathrm{NaOH}) .1 .7 \mathrm{~g}$ of $\mathrm{ZnCl}$ was broken down in $25 \mathrm{ml}$ ethyl alcohol with attractive mixing. In the meantime, $0.9 \mathrm{~g}$ of $\mathrm{NaOH}$ was disintegrated in $25 \mathrm{ml}$ of ethyl alcohol independently. $\mathrm{NaOH}$ arrangement was then included drop astute into $\mathrm{ZnCl}$ solution for 45 minutes with constant magnetic stirring at room temperature. The reaction is allowed to proceed for 20 minutes and then the resultant mixture was sealed and kept undisturbed for 24.0 $\mathrm{h}$ to complete the reaction process. White precipitates were settled after reaction. The solid and solution phases were separated by filtering and washed the same with distilled water 2 times and with diluted HCL 2 times. Precipitated $\mathrm{ZnO}$ powder was then annealed at $80^{\circ} \mathrm{C}$ for $1.0 \mathrm{~h}$ and then crushed for uniformities. The crystallinity behaviour of the prepared sample was investigated by XRD and shown in Figure 1.

\section{Fiber pellets}

Completely dried Water hyacinth fibers used for this experiment were cut into small pieces to form circular pellets of $2.0 \mathrm{~cm}$ in diameter and $0.2 \mathrm{~cm}$ in thickness using a pelletizer. Figure $2 \mathrm{a}$ and Figure $2 \mathrm{~b}$ shows the photograph of raw water hyacinth pellet and plasma treated $\mathrm{ZnO}$ coated pellets respectively.

\section{Plasma treatment}

Initially pellets were kept in a DC glow discharge Ar plasma for different times at a particular experimental condition. Schematic of the experimental set up is shown in Figure 3. The system consisting is of cylindrical shape with $50 \mathrm{~cm}$ in height and $40 \mathrm{~cm}$ in length. Pellets were set 
on a substrate holder inside the chamber before evacuating the system utilizing a diffusion pump backed by a rotary pump. The base pressure has been kept up at $3.0 \times 10^{-6}$ mbar. Ar gas was introduced into the system at a working pressure of $1.7 \times 10^{-1}$ mbar and DC voltage has been applied to make discharge. The discharge current for complete experiment was maintained at 0.4 to $0.6 \mathrm{~A}$ by changing the discharge voltages. The fluxes of ions at low temperature Ar plasma were used to modify the surface chemistry of fiber samples of the order of atomic dimension.
The plasma density and temperature have been measured by an indigenously developed plane Langmuir probe [26] of area $0.28 \mathrm{~cm}^{2}$ and placed close to the target holder. When $350 \mathrm{~V}$ discharge voltage was applied than the plasma electron density and temperature were found to be $n_{e} \sim 10^{14} \mathrm{~m}^{-3}$ and $T_{e}=1.0-3.0 \mathrm{eV}$, respectively and the condition is remain almost same for complete experiment. In a room temperature Water hyacinth pellets have been treated in plasma for different time viz., 5 mins., 15 mins., $30 \mathrm{mins}, 1.0 \mathrm{~h}$ and $1.30 \mathrm{~h}$. respectively and compared the result in this report.

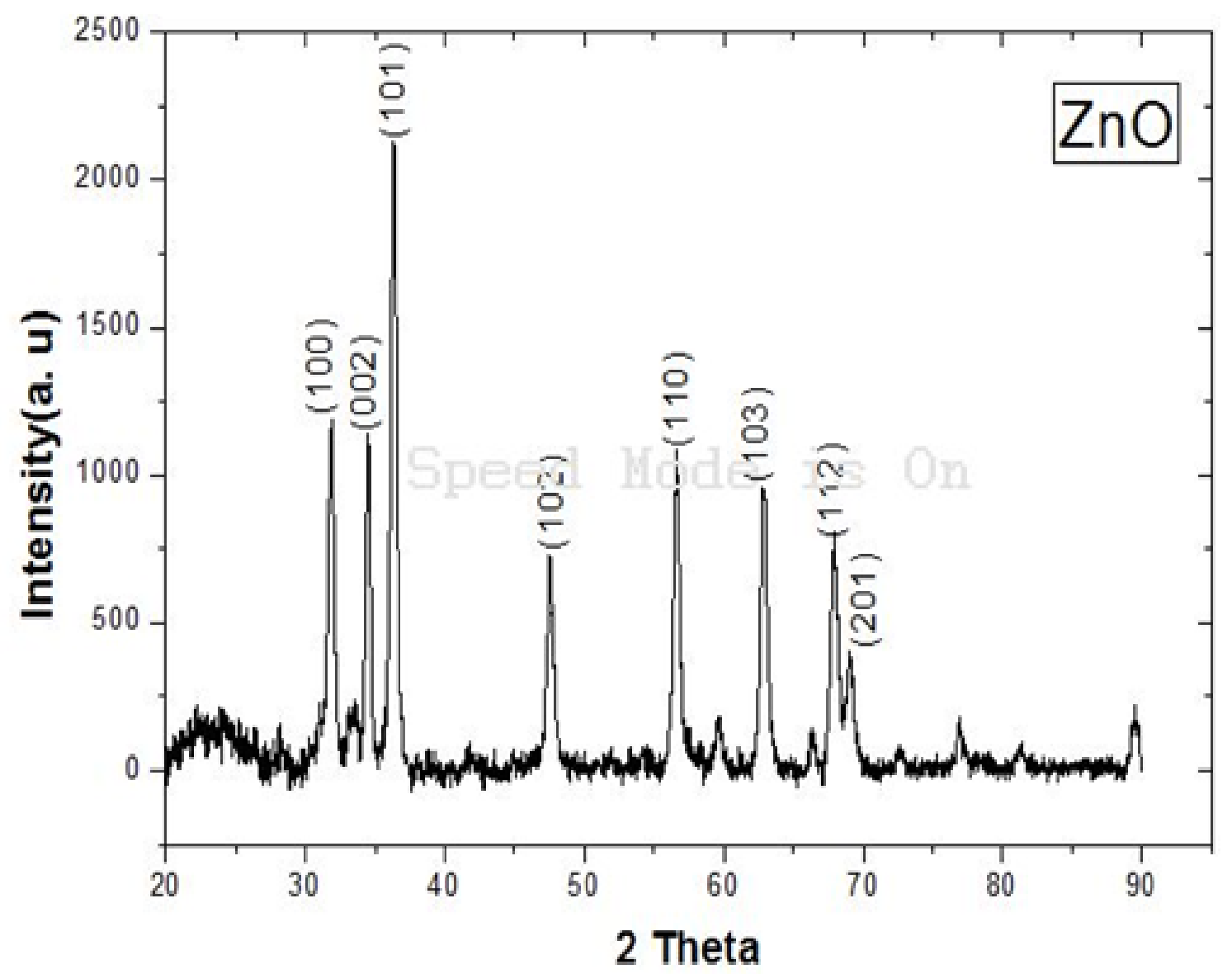

Figure 1. X-ray diffraction pattern of Zinc Oxide $(\mathrm{ZnO})$ nano-particles prepared by chemical synthesis process
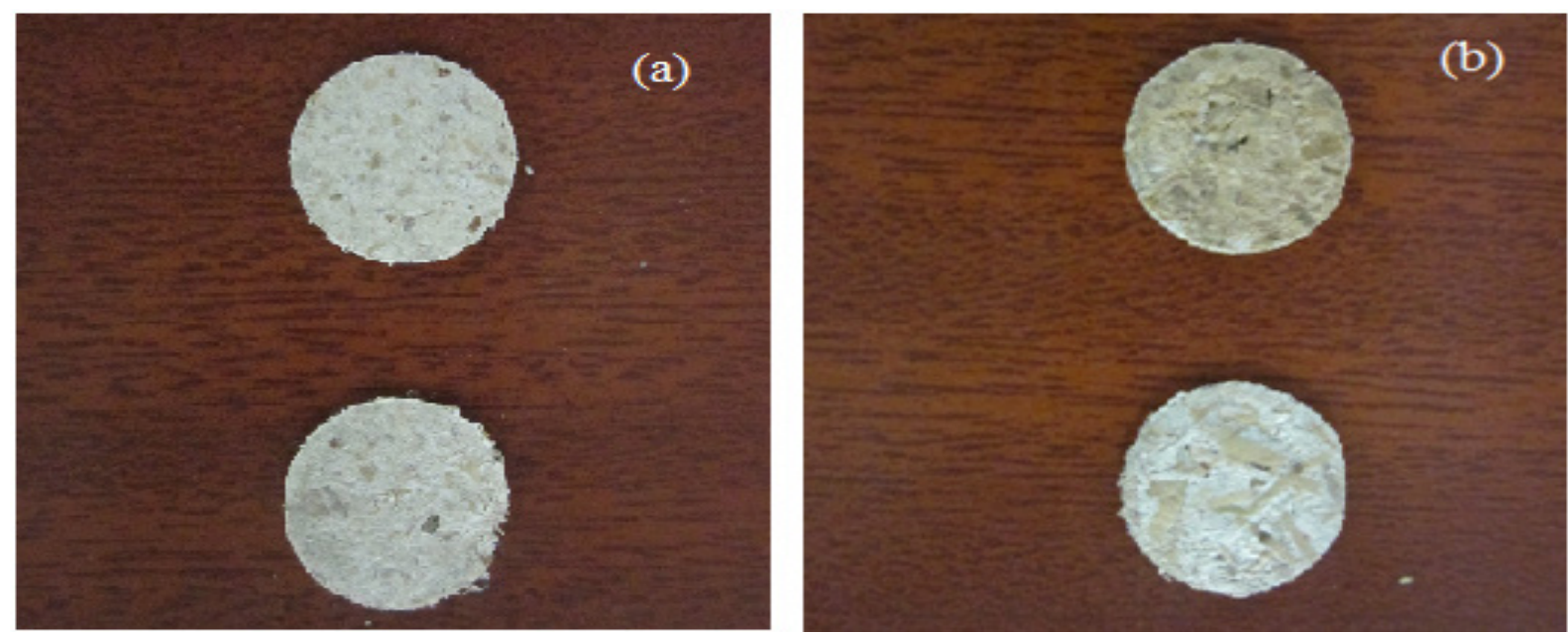

Figure 2. Photograph of a) raw water hyacinth and b) plasma treated $\mathrm{ZnO}$ coated fiber pellets 


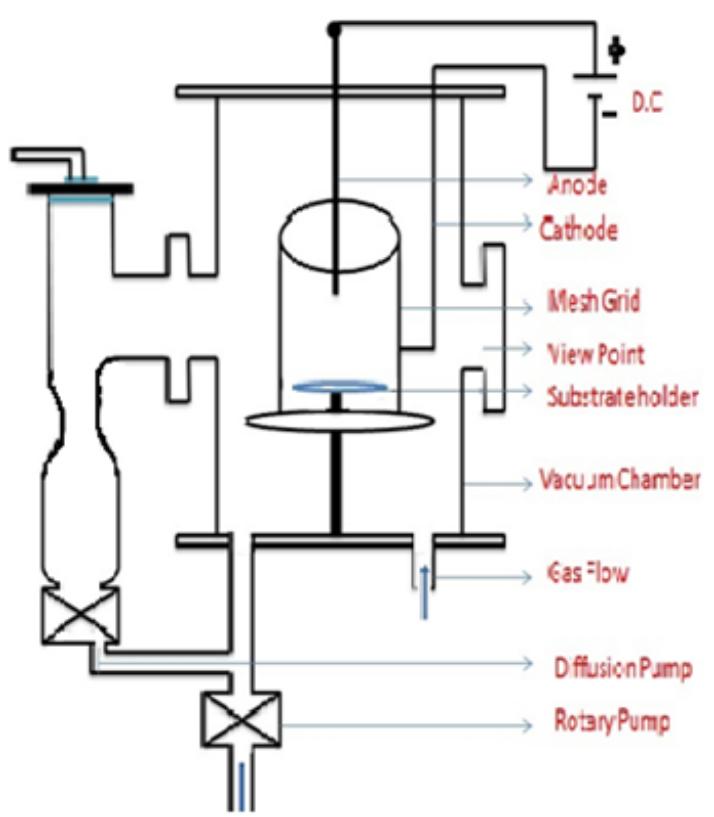

Figure 3. Experimental set up for plasma treatment

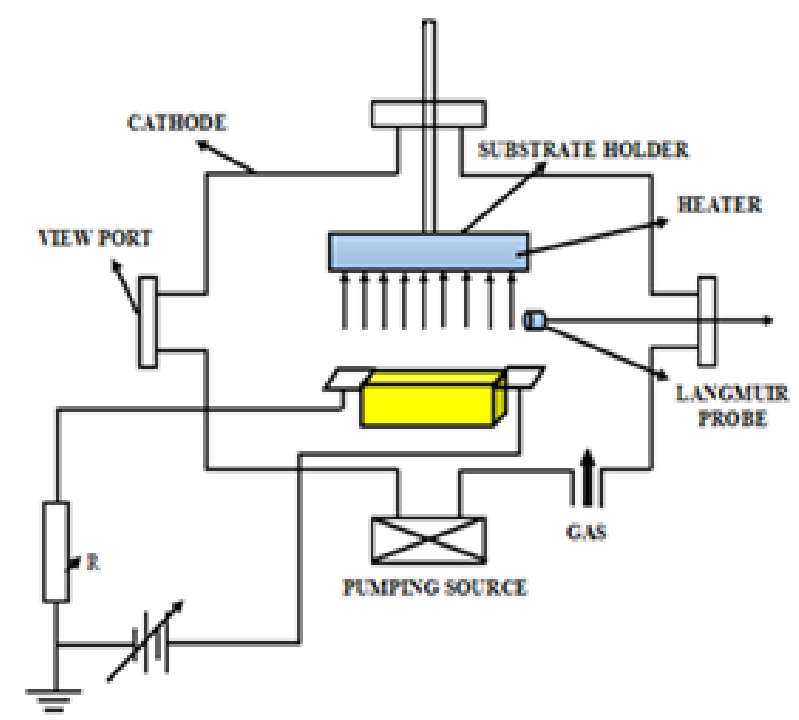

Figure 4. Experimental set up of Thermal Evaporation unit

\section{Thermal evaporation Method}

Plasma treated water hyacinth fiber pellets were then coated with $\mathrm{ZnO}$ nano particles for its surface treatment. A Thermal evaporation system has been used to coat the $\mathrm{ZnO}$ film in such a way that the bulk property of fibers remains almost same. Figure 4 shows the Schematic outline of the thermal evaporation system. Evaporation was carried out in round and hollow load with $40 \mathrm{~cm}$ in stature and $30 \mathrm{~cm}$ in distance across. Plasma treated Water hyacinth fibers were kept on a substrate holder inside the system before evacuating the system to $10^{-6} \mathrm{mbar}$. Inside the system the $\mathrm{ZnO}$ nano powders were positioned on a molybdenum boat. Admittance and density of the experimental conditions related to $\mathrm{ZnO}$ have been fixed as $15.88(\Omega)$ and $5.61\left(\mathrm{~g} / \mathrm{cm}^{3}\right)$ in order to achieve uniform coating throughout the surface. When the current applied to the boat was set between $5.0 \mathrm{~A}$ to $8.0 \mathrm{~A}$, the nano powder starts to evaporate and deposited on the water hyacinth fiber. The process time has been fixed at 5.0 minutes for all plasma treated fibers.

\section{Characterization}

Thermo gravimetric analysis (TGA)

TGA estimations have been performed utilizing a (TGA, TG/DTA 7200 EXSTAR instrument) warm analyzer. A warming rate of $15^{\circ} \mathrm{C} / \mathrm{min}$ and an specimen weight of 3-5 $\mathrm{mg}$ in an aluminum cauldron with a stick opening have been utilized as a part of a nitrogen air $(50 \mathrm{ml} / \mathrm{min})$.

$X$-ray diffraction (XRD)

XRD pattern for synthesized $\mathrm{ZnO}$ powder, raw water hyacinth fiber and plasma treated $\mathrm{ZnO}$ coated fibers have been done at various experimental conditions. The dispersing $d$ of an arrangement of planes in a crystal is identified with the Bragg edge $\theta$, and the wavelength $\lambda$, considering the Bragg condition. Contingent upon the dispersing of the non-treated, the treated $d_{s}$ (strained) fiber $d_{i}$ (unstressed) the miniaturized scale strain in the particles in the heading ordinary to the diffracting plane given by [27],

$$
\zeta=\frac{\Delta d}{d}=\left(\frac{d_{s}-d_{i}}{d_{i}}\right) .
$$

Here, $d_{s}<d_{i}$ or $d_{s}>d_{i}$, then $\Delta d / d$ is positive or negative that shows whether the lingering stress is malleable or constricting in the surface, respectively. The estimation of $\Delta d / d$ must be understood to know its extreme ductile strain or greatest constricting strain [28]. On the other hand, the crystalline size of the examples have been ascertained by Scherrer formula [29],

$$
T=\frac{k \lambda}{\beta \cos \theta}
$$

Where, $\beta$ is the line broadening at half the maximum intensity (FWHM), $k$ is a dimensionless shape factor $(=0.94), \theta$ the Bragg angle and $\lambda$ the X-ray wavelength [30].

The dislocation density is a measure of how many number of dislocations present in a unit volume of a crystalline material and it is expressed as,

$$
\delta=\frac{1}{D^{2}}
$$

Again, strain is defined as the relative change in the position of points within a crystal that has undergone deformation i.e., (Dislocation in crystal lattice)

$$
\varepsilon=\frac{\beta \cos \theta}{4} \text {. }
$$

The crystallinity index (C.I) of water hyacinth fiber by height has been done [31] using a computing stratergy as follows,

$$
C . I(\%)=\frac{h_{c}}{h_{t}} X 10
$$

Where $h_{c}=$ height of primary crystalline peak $h_{t}=$ total height of primary crystalline peak.

Contact angle (Goniometer)

Contact Angle measurement was carried out by Rams hart instrument (Co.Model-250). It is used to measure the angle between the outline tangents of a drop deposited on a fibers with the corresponding surface of the same. 
Fourier transform infrared (ATR-FTIR)

The untreated and plasma treated $\mathrm{ZnO}$ coated fibers were examined by ATR- FTIR spectroscopy. Roomtemperature ATR-FTIR spectra have been registered using a Nicolet ATR-FTIR Spectroscopy (IS10). The spectra have been smoothened with a consistent smooth element for correlation.

Morphology studies

Scanning electron microscope (SEM) have been carried out for the Surface monograph of samples using a (HRSEM, Hitachi S-4800 type) magnifying instrument.

Energy dispersive $X$-ray $(E D A X)$

To know the elemental information's of raw and plasma treated $\mathrm{ZnO}$ coated fibers EDAX analysis has been performed. The measurement has been conducted by the Hitachi (model No. S-4800 SEM) furnished with a cathode and attractive focal points to make and center a light emission for basic examination.

Test for water absorption

The fibers was set up at room temperature lastly adapted for $12.0 \mathrm{~h}$ for assurance of final weight $\left(W_{l}\right)$. Then the fiber were submerged in refined water for $24.0 \mathrm{~h}$, then dried to get the final saturated weight $\left(W_{2}\right)$ was also estimated for the sample [32]. Percentage of absorption of water was Figureured with the formula

$$
\mathrm{W}(\%)=\frac{\left(w_{2}-w_{1}\right)}{w_{1}} \mathrm{X} 100 .
$$

\section{Results and Discussions}

\section{Thermo gravimetrical analysis of the samples}

The TGA/DTA analysis was done for the untreated and plasma treated $\mathrm{ZnO}$ coated fiber. Figure 5 shows the TGA curve of untreated and plasma treated nano-particle coated fibers. In case of raw fiber, initial weight loss was at around $76.5^{\circ} \mathrm{C}$ and can be attributed to removal of entrapped water and further weight loss at $227.4^{\circ} \mathrm{C}$ [33]. More weight loss onberved at temperatures $354.2^{\circ} \mathrm{C}$, $573.7^{\circ} \mathrm{C}$ and $751.8^{\circ} \mathrm{C}$, respectively as shown in Figure. 5a. The degradation of cellulose stared at these temperatures leading to the development of char. Hence, it was observed that the water content of the plasma treated and $\mathrm{ZnO}$ coated fiber is getting reduced than the raw fibers due to surface modification of the same. In case of $1.30 \mathrm{~h}$ plasma treated $\mathrm{ZnO}$ coated fiber, the initial weight loss took place at around $87.7^{\circ} \mathrm{C}$, which is more than the raw fiber and occurs further at $238.6^{\circ} \mathrm{C}, 357.7^{\circ} \mathrm{C}, 634.5^{\circ} \mathrm{C}$ and $754.2^{\circ} \mathrm{C}$, respectively as shown in Figure $5 \mathrm{c}$. Due to considerable decrease in moisture content in treated fibers, the first peak of TGA curve gets almost disappeared. This small increase in temperature in its weight loss processes reflects the loss of moisture content of the plasma treated $\mathrm{ZnO}$ coated fibers. Similar result was been observed in 1.0 $\mathrm{h}$ treated $\mathrm{ZnO}$ coated fibers and shown in Figure $5 \mathrm{~b}$. The residual char left at $800^{\circ} \mathrm{C}$ has increased to $79 \%$ for 1.30 $\mathrm{h}$ plasma treated $\mathrm{ZnO}$ coated fibers when compared to raw fibers $73 \%$. This demonstrates the rate $(\%)$ increases of cellulose substance in treated fibers. Fibers are then exposed to air atmosphere for $24.0 \mathrm{~h}$, which confirms the variation of moisture content on plasma tretaed fibers due to the plasma surface modificationon fibers.

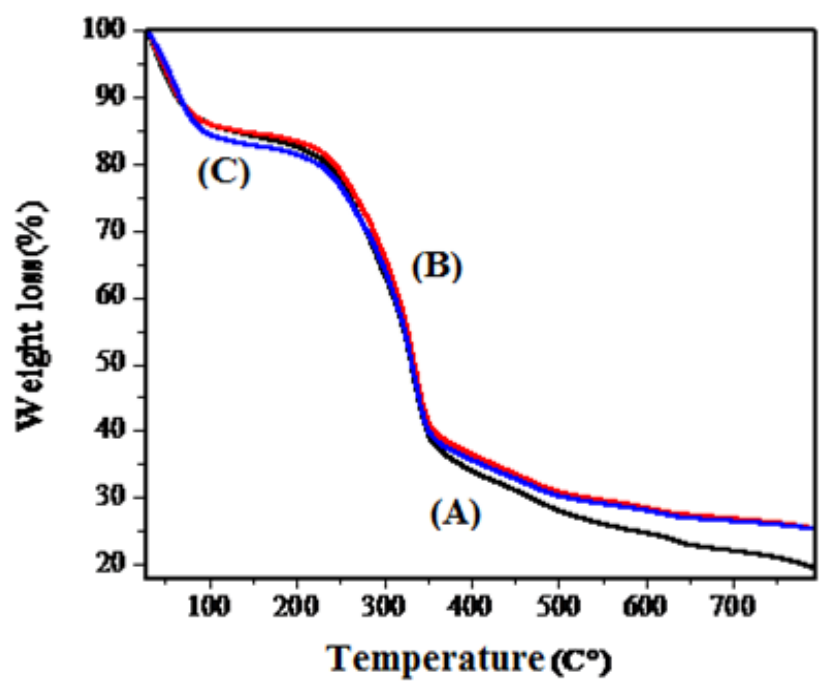

Figure 5. TGA curve of (A) untreated fiber, (B) $1.0 \mathrm{~h}$ plasma treated coated fiber and (C) $1.30 \mathrm{~h}$ plasma treated coated fiber.

\section{$X$-ray diffraction Analysis}

The room temperature XRD pattern of untreated, $1.0 \mathrm{~h}$ and $1.30 \mathrm{~h}$ plasma treated $\mathrm{ZnO}$ coated fibers are shown in Figure 6 respectively. The pattern clearly shows the increase in $d$-spacing due to the plasma treated $\mathrm{ZnO}$ coated fibers. The micro stress and \% of crystallinity [34] were calculated from the diffractometer and are presented in Table 1 . Obviously the line broadening at half of the maximum intensity (FWHM) decreases for plasma treated coated $\mathrm{ZnO}$ fibers. The level of crystallinity Index (CrI\%) utilizing the Segal exact strategy has additionally increased after plasma treated coated $\mathrm{ZnO}$ fiber. This increase in crystallinity with plasma treated coated $\mathrm{ZnO}$ fibers also confirms the increase in durability of the fiber as given by TGA analysis.

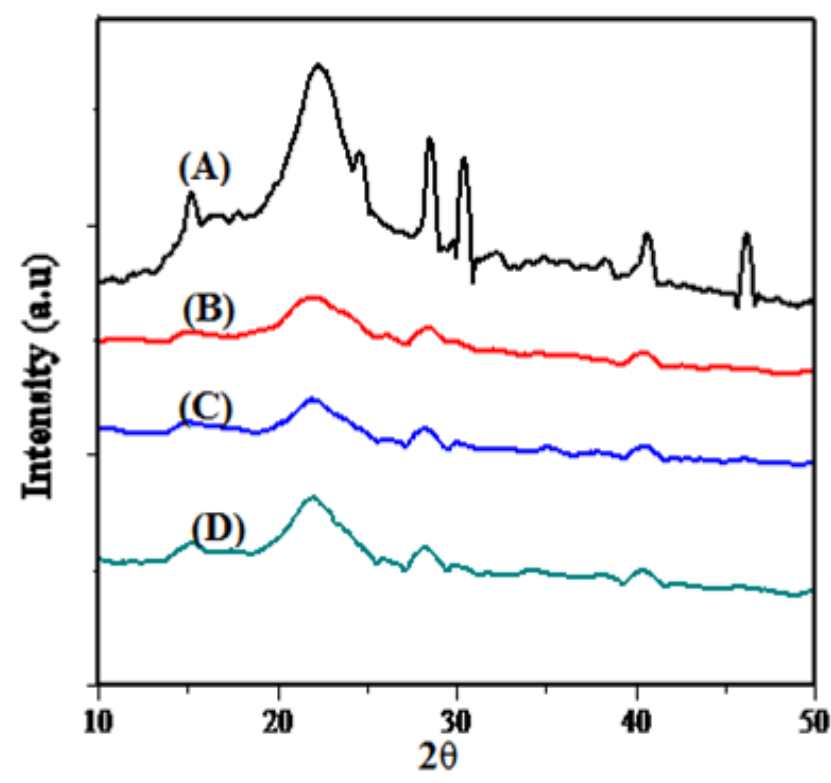

Figure 6. XRD pattern of (A) Raw fiber (B) 30 minutes plasma treated coated fiber and (C) $1 \mathrm{~h}$ plasma treated coated fiber (D) $1.30 \mathrm{~h}$ plasma treated coated fiber 
Table 1. The observed and computed parameters from the XRD Pattern

\begin{tabular}{|c|c|c|c|c|}
\hline Parameters & Raw fiber & $\begin{array}{c}30 \text { mins plasma treated coated } \\
\text { ZnO }\end{array}$ & $\begin{array}{c}1 \text { h plasma treated } \\
\text { coated } \mathrm{ZnO}\end{array}$ & $\begin{array}{l}1.30 \text { h plasma treated } \\
\text { coated } \mathrm{ZnO} \\
\end{array}$ \\
\hline 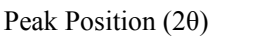 & 22.18 & 21.40 & 21.97 & 21.24 \\
\hline 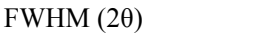 & 2.30 & 1.96 & 1.57 & 1.50 \\
\hline $\mathrm{d}(\AA)$ & 5.82 & 5.92 & 4.24 & 5.93 \\
\hline Crystal size $(\AA)$ & 6.4 & 7.5 & 9.3 & 9.8 \\
\hline Strain $(\varepsilon)$ & 0.56 & 0.48 & 0.38 & 0.36 \\
\hline CrI (\%) & 46.67 & 48.14 & 52.17 & 55.88 \\
\hline Dislocation density $(\delta)$ & 2.44 & 1.77 & 1.15 & 1.04 \\
\hline
\end{tabular}

\section{Wettability}

Wettability of the treated and untreated fiber surface has been characterized by the goniometer static contact angle. It relies upon components like roughness, cleanliness of the surface and surface preparation processes. At the point when the fluid wets the surface and the comparing estimation of static contact edge is $<90^{\circ}$ then the surface is referred as hydrophilic surface and when it is $>90^{\circ}$ yet $<180^{\circ}$, then it is referred to as hydrophobic and water repellent surfaces.

Image of Contact angle measurement of untreated and plasma treated $\mathrm{ZnO}$ coated fibers with water drop using Goniometer is shown in Figure. 7. The measured data shows that-raw fibers it is $52.7^{\circ} \mathrm{C}$ while for 5 minutes, 30 minutes and $1.0 \mathrm{~h}$ plasma treated $\mathrm{ZnO}$ coated fibers it becomes $57.4^{\circ} \mathrm{C}, 98.2^{\circ} \mathrm{C}$ and $99.2^{\circ} \mathrm{C}$, respectively (obtuse angle), which clearly signifies the hydrophobic nature of treated sample. Hence, the surface will act as more water repellent surface [35].
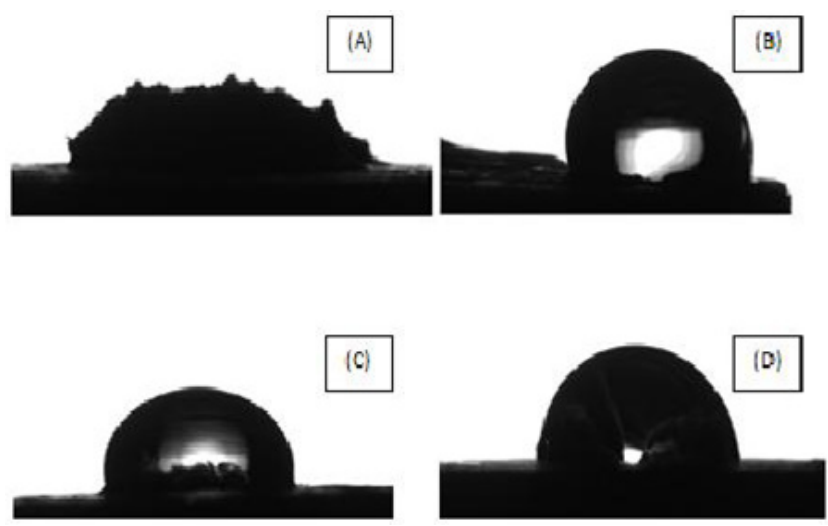

Figure 7. Contact angle measurement of raw and plasma treated Zinc Oxide coated fibers with water drop have been measured using images of Goniometer of (A) untreated (B) $5 \mathrm{mins}$ (C) $30 \mathrm{mins}$ and (D) $1.0 \mathrm{~h}$ plasma treated coated fiber

\section{Chemical composition analysis}

To obtain information about chemical changes that takes place within the fiber due to plasma treatment as well as $\mathrm{ZnO}$ coating on it, the Fourier transform infra-red (FTIR) spectra has been done and shown in Figure. 8. Here, $3327 \mathrm{~cm}^{-1}$ band has been attributed to $\mathrm{OH}$ Stretching and shown almost similar absorbance intensity proportion for the raw and plasma treated coated $\mathrm{ZnO}$ fiber because of $\alpha$-cellulose contents. The $2925 \mathrm{~cm}^{-1}$ is relegated to $\mathrm{CH}_{2}$ stretching in methyl and methylene group. The band near $1731 \mathrm{~cm}^{-1}$ is doled out to the $\mathrm{C}=\mathrm{O}$ stretching of the carboxyl and acetyl amasses in hemicelluloses of the fiber. The band $1645 \mathrm{~cm}^{-1}$ is connected with ingestion of water.

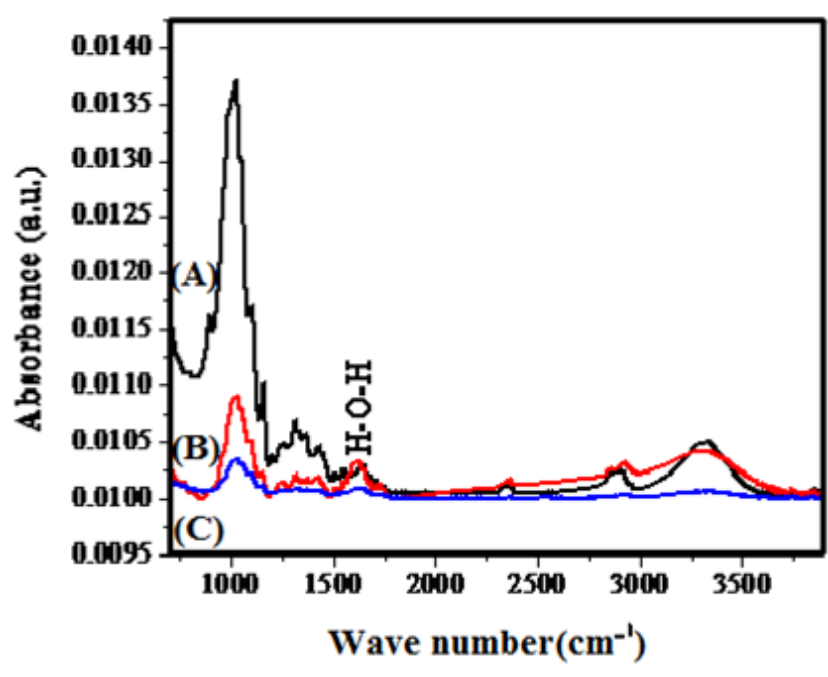

Figure 8. ATR-FTIR spectra of (A) untreated fiber (B) 30 mins and (C) $1.0 \mathrm{~h}$ plasma treated Zinc Oxide coated fiber

This absolute intensity of this band i.e. corresponding to $1645 \mathrm{~cm}^{-1}$ is get reduced in case of $1.0 \mathrm{~h}$ and $1.3 \mathrm{~h}$ plasma treated $\mathrm{ZnO}$ coated fibers, respectively due to reduction moisture content from the fibers. The absorbance at 1437 $\mathrm{cm}^{-1}$ is ascribed to $\mathrm{CH}_{2}$ twisting (topsy-turvy) in lignin. This retention reduces after plasma treatment of $1.0 \mathrm{~h}$ and which might be because of loss of lignin. The medium band at $1249 \mathrm{~cm}^{-1}$ is attributed to C-O-C asymmetric stretching is also reduces after plasma treatment due to acetyl group removal. Then again, the band at $1028 \mathrm{~cm}^{-1}$, is normally assigned to C-O Stretching.

\section{Surface Morphology of samples}

Scanning electron microscope (SEM) micrographs has been taken to understand the alteration of the surface morphology of fibers in all experimental conditions as mentioned already. Figure 9 shows the microstructures of untreated and $1.0 \mathrm{~h}, 1.30 \mathrm{~h}$ respectively.

Monograph clearly indicates the relatively rough surface of raw fibers without any multi-cellular nature. Thus plasma treated coated $\mathrm{ZnO}$ fibers results significant changes in its morphology on account of the evacuation of lignin and hemicelluloses and in addition other structural impacts with respect to treatment time. $1.30 \mathrm{~h}$ plasma treated fibers shows more smoothness, compared to the raw and $1.0 \mathrm{~h}$ plasma treated $\mathrm{ZnO}$ coated fiber.

To comprehend the surface properties more quantitatively, open source programming Gwyddion (adaptation 2.35) has been utilized for the picture preparing of the SEM images [36]. Figure. 9 demonstrates the 3-Dimensional perception of the fiber surface at 
various test conditions utilizing programming. Plot of the same has been made utilizing the Interactive 3-D surface plot module of ImageJ [36]. Figure 10 (a), (b) and (c) demonstrates the surface topology of untreated, $1.0 \mathrm{~h}$ and $1.30 \mathrm{~h}$ plasma treated $\mathrm{ZnO}$ coated fiber, respectively. The
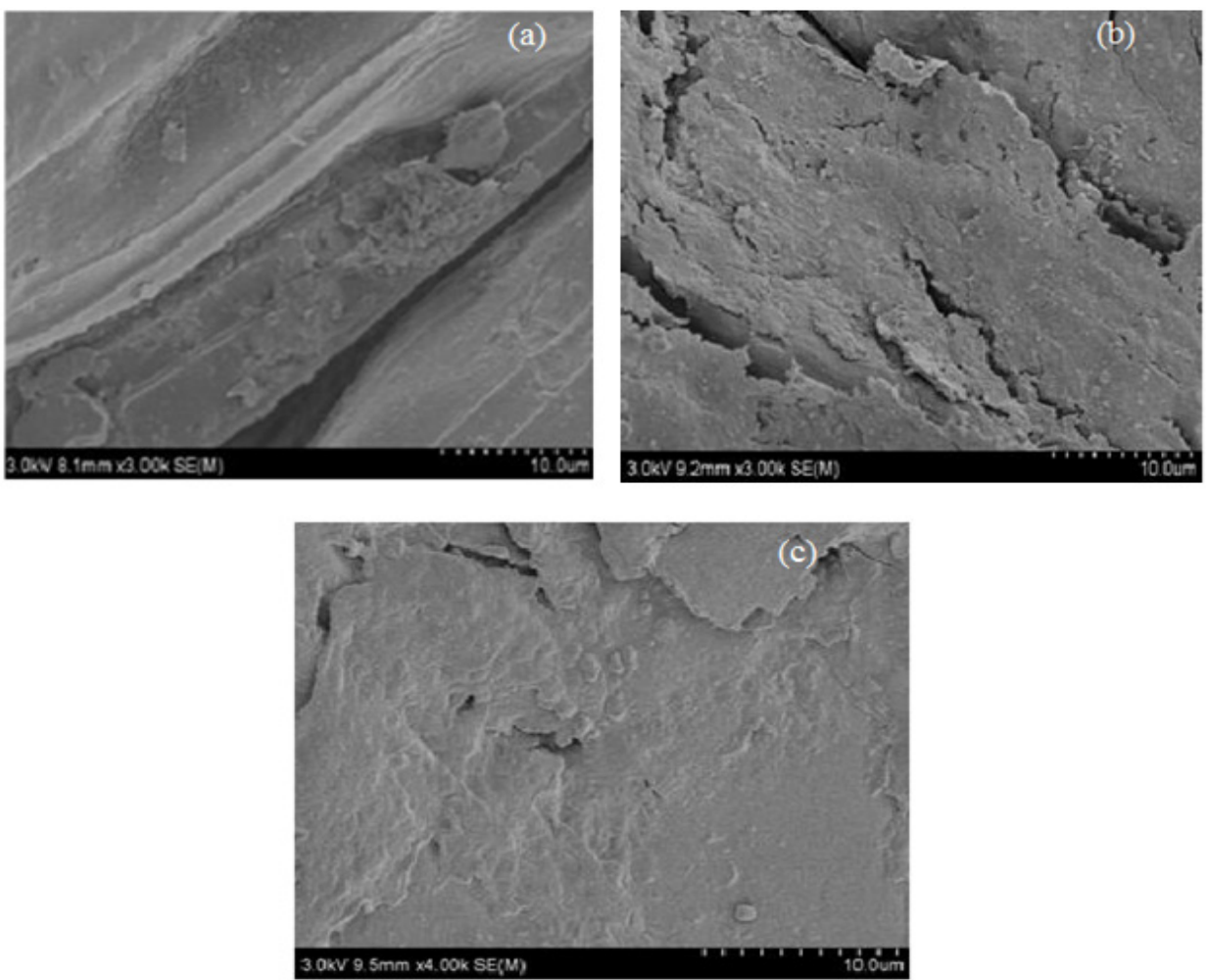

Figure 9. Scanning Electron microscope Image of (A) untreated fiber (B) $1.0 \mathrm{~h}$ plasma treated Zinc Oxide coated fiber (C) $1.30 \mathrm{~h}$ plasma treated Zinc Oxide coated fiber
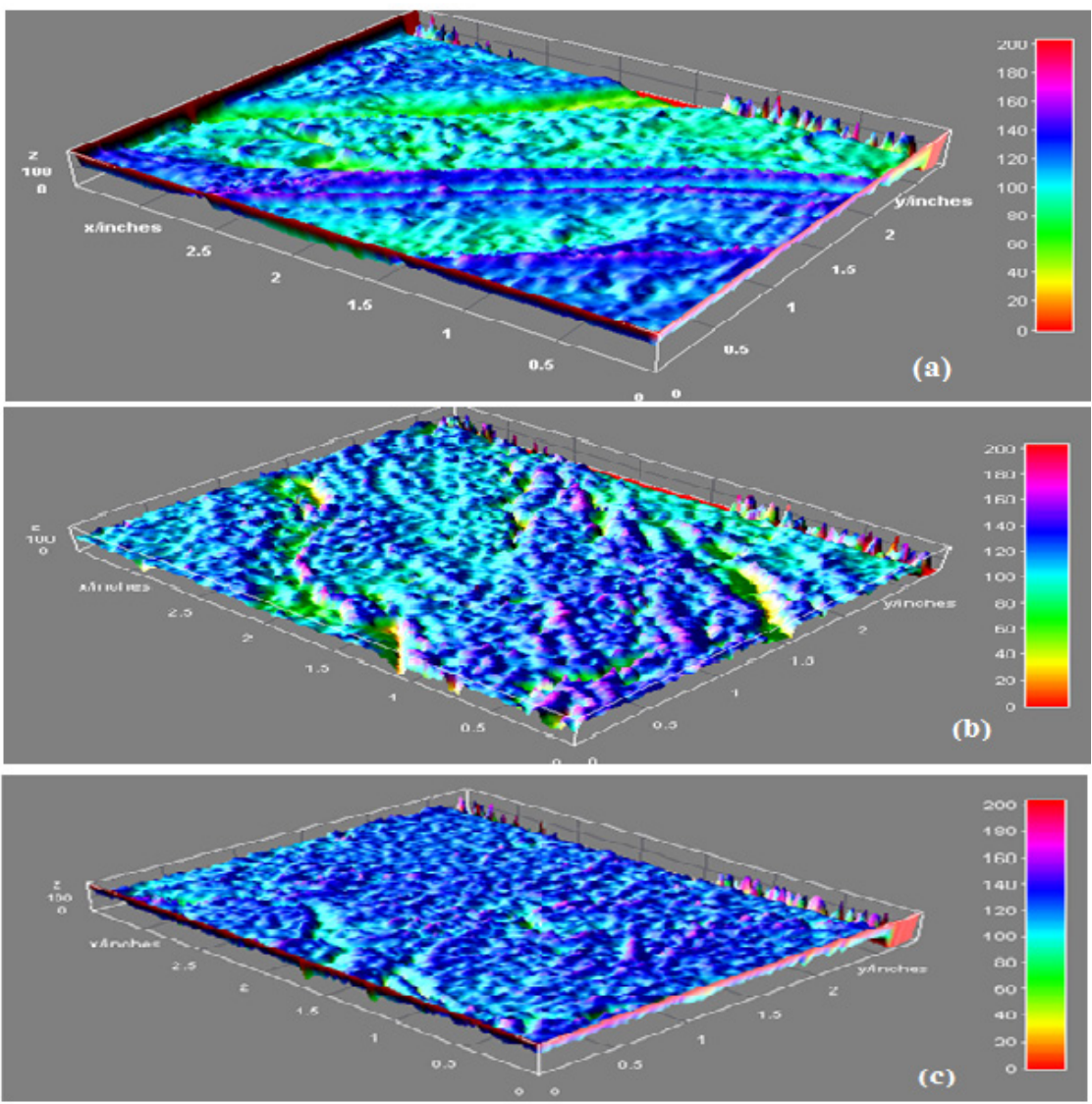

Figure 10. Morphology of (A) untreated fiber (B) $1.0 \mathrm{~h}$ plasma treated coated Zinc Oxide fiber (C) $1.30 \mathrm{~h}$ plasma treated coated Zinc Oxide fiber 
Table 2. Surface roughness of untreated and plasma treated $\mathrm{ZnO}$ coated fibers.

\begin{tabular}{|c|c|c|c|c|}
\hline Parameters & Roughness (nm) & Root Mean Square (nm) & Skewness & Kurtosis \\
\hline Untreated Fiber & 96.7 & 152.8 & 0.136 & 4.84 \\
\hline 1.0h plasma treated and $\mathrm{ZnO}$ coated fiber & 102.0 & 165.5 & 0.137 & 4.01 \\
\hline 1.30h plasma treated and $\mathrm{ZnO}$ coated fiber & 96.4 & 185.4 & 0.497 & 3.77 \\
\hline
\end{tabular}

Average roughness and arithmetic mean with respect to plasma treatment time has been calculated and shown in Table 2. This indicates that surfaces do not lose its individual property due to plasma treatment. These differences are not large enough to be appreciated in the SEM images of the respective surfaces, which is quite clear in the $3 \mathrm{D}$ image of the surface Figure. 10. The Skewness of the surface stature circulation mostly remains in the positive administration and thus keeps up the symmetry.

\section{Wavelet Transformation}

Daubechies wavelet of order 8 (MATLAB) has been used as the basic function for the calculations. Figure 11 shows the distinctive frequency parts (wavelet packets) acquired by MRSD of the surface profile of SEM images. The progressions in $A_{n}^{k}$ and $D_{n}^{k}$ under eight distinct resolutions are examined for both treated and untreated samples. It has been observed that the amplitudes of $A^{k}{ }_{n}$ and $D_{n}^{k}$ are smaller for plasma treated $\mathrm{ZnO}$ coated fiber when compared with the raw fibers, thus it is revealing that the roughness is decreasing in the surface of the fibres.

The average energies (entropy) at different frequency levels for both raw and plasma treated $\mathrm{ZnO}$ coated fiber profiles are indicated in Figure 12. The energies are calculated by using entropy condition is shown in Eq (4) for the decomposed wavelet bundle at position " 3 " has been taken as a source of perspective for all image profiles.

\section{Raw fiber}

1

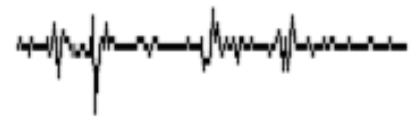

2

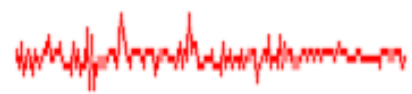

3

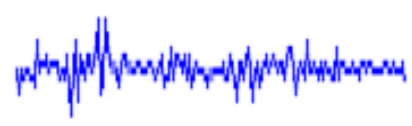

4

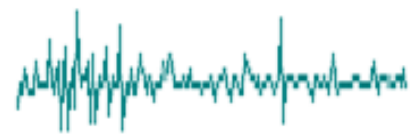

5

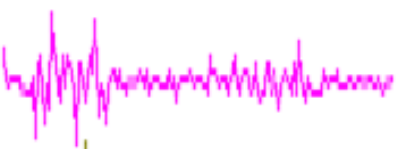

6

7

8

9

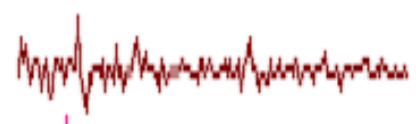
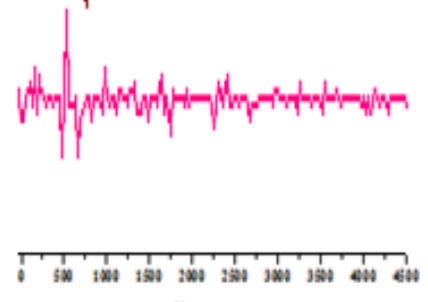

\section{1h Plasma treated} ZnO coated

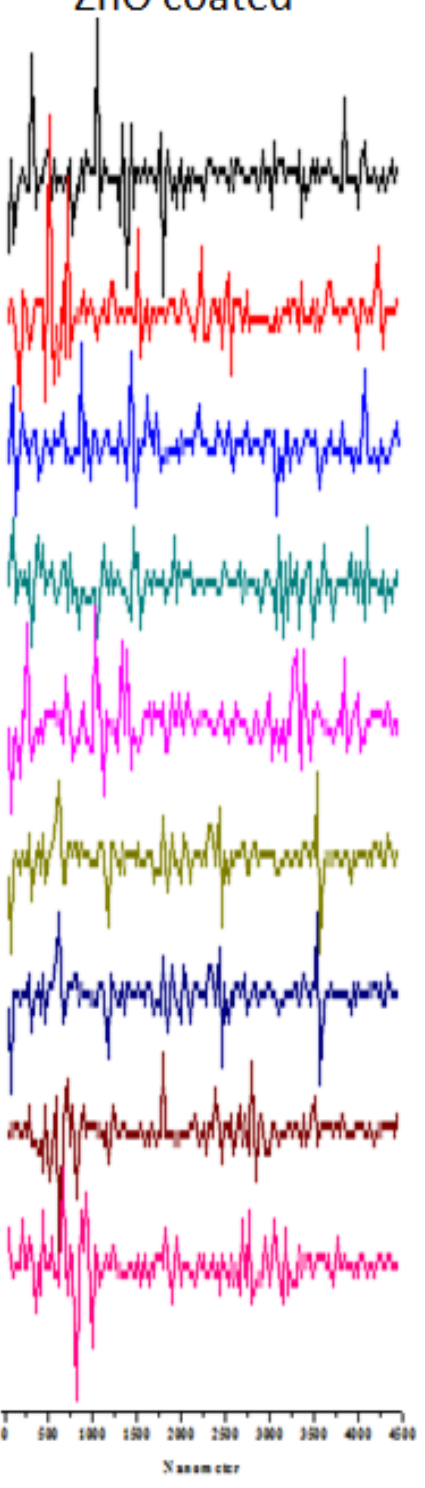

\subsection{0h plasma treated ZnO coated fiber}
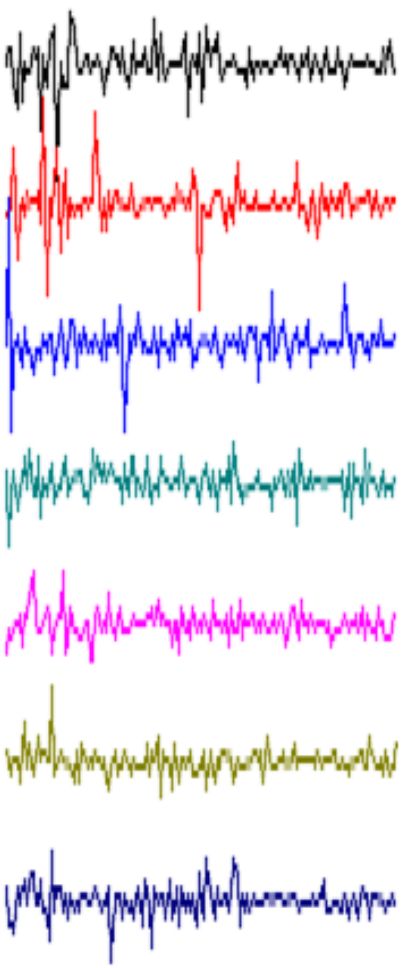

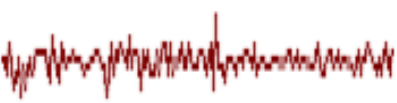

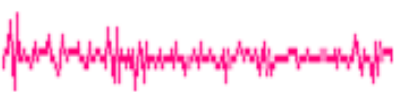

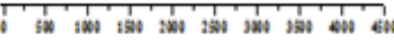
Yaum tar

Figure 11. MRSD of the surface profiles of SEM images for $\mathrm{ZnO}$ coated water hyacinth fiber before and after plasma treatment 


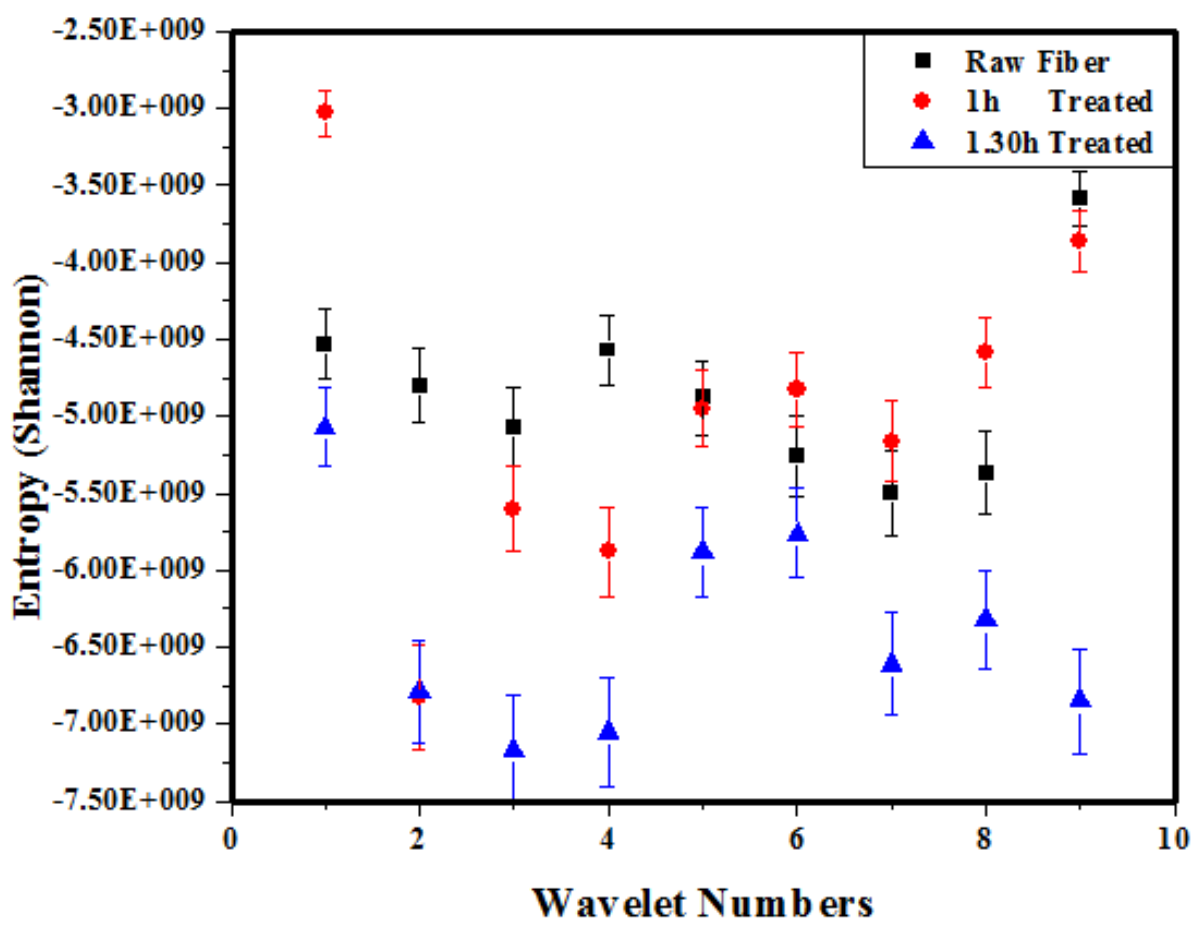

Figure 12. Average energy (entropy) of different frequency components (wavelet packet) at the third (final) stage of decomposition of signal profiles of $\mathrm{ZnO}$ Coated swater hyacinth fiber before and after plasma treatment

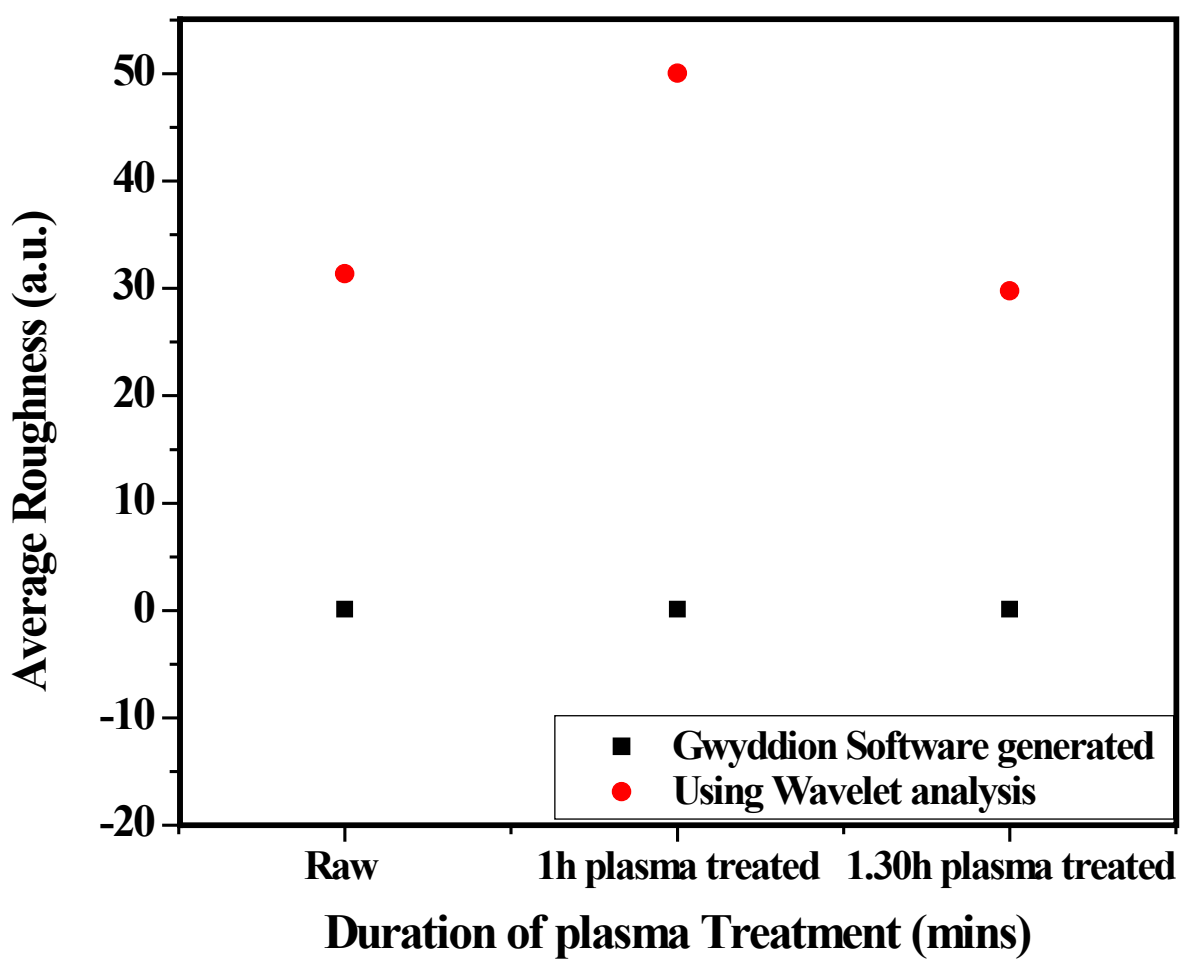

Figure 13. Roughness Comparison between Software generated image and using wavelet analysis

Figure 12 is exhibiting the decreasing pattern of energy in the element bundles. For raw fibre and $1.0 \mathrm{~h}$ treated fibre, it is almost identical. But it is clearly visible that energy is falling and it describes the roughness is decreasing with $1.3 \mathrm{~h}$ treatment.

The calculated values of surface roughness (peak-tovalley height differences), averaged over 10 different profiles for each membrane sample. This gives direct roughness of the surface at respective positions directly from the decomposed wavelet packets. The variation in the rms values of the wavelet coefficients with respect to the frequency range provides energy or entropy associated with decomposed packet whose value has also been calculated. The normal energies (entropy) at various frequency levels for both treated and untreated fiber.

There is no much difference in the entropy for raw and $1.0 \mathrm{~h}$ treated fiber. The differences in entropy of all samples lies within the value of 3 , i.e., less than $5 \%$ of the overall values. From the decomposed signal data, the average roughness $\left(R_{a}\right)$ has been calculated for all wavelet 
packets using Eq (1) and the values are compared with the average roughness values calculated from the Gwyddion software which is shown in Figure 13. Quantitatively the roughness values are remaining similar with particular method of calculation.

$$
\mathrm{R}_{\mathrm{a}}=\frac{1}{n} \sum_{i=1}^{n}\left|y_{i}\right|
$$

\section{Elemental analysis}

Table 3 shows EDAX analysis of the atomic weight $\%$ of untreated, $1.0 \mathrm{~h}$ and $1.30 \mathrm{~h}$ plasma treated $\mathrm{ZnO}$ coated fiber, respectively. Since $\mathrm{ZnO}$ is coated on fibers, the $1.99 \%$ and $7.36 \% \mathrm{Zn}$ element have been identified for 1.0 $\mathrm{h}$ and $1.3 \mathrm{~h}$ plasma treated $\mathrm{ZnO}$ coated fiber which was not identified in the untreated fiber. Light elements like $C$, $\mathrm{O}_{2}$ are identified mostly with few other elements like $\mathrm{Mn}$, $\mathrm{Cl}, \mathrm{S}, \mathrm{Ca}$ and $\mathrm{K}$.

\section{Water absorption test}

Water absorption examination of the untreated and plasma treated $\mathrm{ZnO}$ coated fibers were studied using water absorption or water uptake (\%) processes.

The moisture Absorption of the fibers was reaped by hydrophobicity Measurements. The rate of water assimilation (W) was computed by asn experimental equation for untreated and plasma regarded $\mathrm{ZnO}$ coated fibers as takes after:

$$
W=\frac{w_{2}-w_{1}}{w_{1}} \times 100
$$

where the dry weight $\left(w_{l}\right)$ and submerged weight $\left(w_{2}\right)$ were measured to Figureure the water take-up of the untreated and plasma treated $\mathrm{ZnO}$ coated fibers. The dry weight, drenched weight, and water take-up rate for untreated fiber were $0.0075,0.010$, and $33.3 \%$, separately.

Similar parameters for fibers treated for 30 mins were $0.007,0.009$, and $28.5 \%$, proceeded by $1.0 \mathrm{~h}$ plasma treated $\mathrm{ZnO}$ coatedd fiber and $1.3 \mathrm{~h}$ plasma treated $\mathrm{ZnO}$ coated fiber is $26.3 \%$ and $12.5 \%$ repectively. The water take-up rates of untreated and treated fibers demonstrate the hydrophobicity of the plasma treated fibers. It demonstrates a decreasing in water take-up ability of plasma treated $\mathrm{ZnO}$ coated fibers. This might be because of the impact of hemicelluloses which are attributed to the demolition and breaking of hydrogen bonds.

Table 3. Atomic weight $\%$ of raw fiber and plasma treated $\mathrm{ZnO}$ coated fibers

\begin{tabular}{cccc}
\hline Elements & Raw Fiber(\%) & $\begin{array}{c}\text { 1h plasma treated and ZnO coated } \\
\text { Water hyacinth fiber(\%) }\end{array}$ & $\begin{array}{c}\text { 1.30h plasma treated and ZnO } \\
\text { coated Water hyacinth fiber(\%) }\end{array}$ \\
\hline Carbon & 66.33 & 54.77 \\
Oxygen & 31.19 & 40.51 & 0 \\
Magnese & 0.41 & 0.54 & 0 \\
Clorine & 0.78 & 0 & 0.73 \\
Potassium & 1.01 & 0.47 \\
Calcium & 0.29 & 0 \\
Sulphur & 0 & 1.99 \\
Zinc & 0 & 8.36 \\
\hline
\end{tabular}

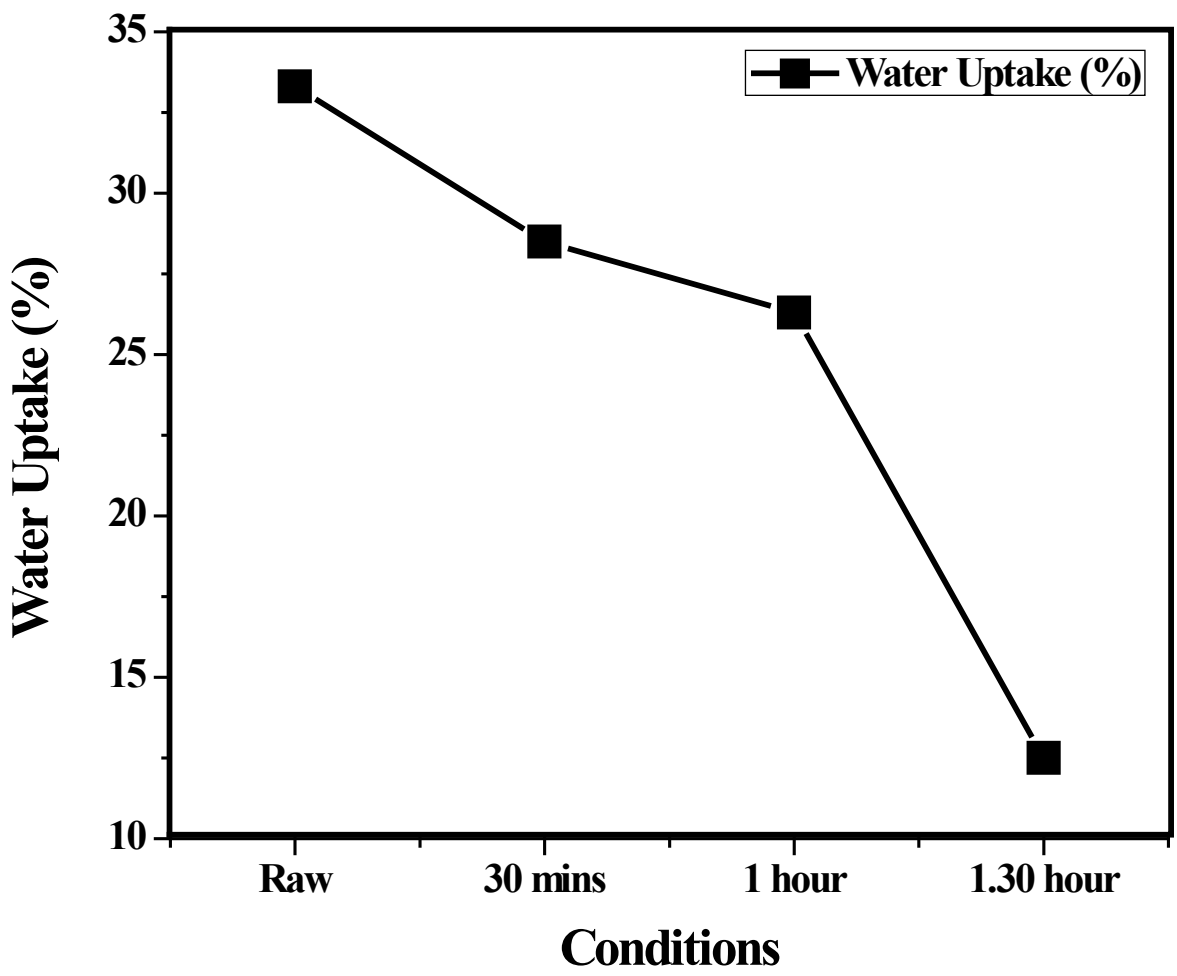

Figure 14. Water absorption analysis of raw and plasma treated $\mathrm{ZnO}$ coated water hyacinth fiber 
Figure 14 assures the hydrophobhic nature of plasma treated $\mathrm{ZnO}$ coated fibers under various experimental conditions. The contact angle goniometer analysis also verifies the hydrophobic nature and hereby we assure that the fibers become more hydrophohic after plasma treatment and coating of $\mathrm{ZnO}$.

\section{Conclusions}

In this study both plasma and nano-technology have been used to increase the water repellent capacity of water hyacinth natural fibers. Pallets of water hyacinth fibers have been treated in plasma for different time and further coated with synthesized $\mathrm{ZnO}$ nano-particles to increase the hydrophobicity. TGA/DTA analysis of the plasma treated $\mathrm{ZnO}$ coating fibers clearly shows the improvement of thermal stability the treated fibres with respect to the untreated fibers. X-ray analysis of the treated sample also signifies the increase of crystallinity index of the fiber and which can be correlated with the water repellent. The SEM-EDAX analysis shows the abrupt change in surface morphology of the plasma treated $\mathrm{ZnO}$ coated fibers compared to untreated fiber and the elemental composition of both raw and plasma treated $\mathrm{ZnO}$ coated fiber respectively. In addition WT method with the MRSD algorithm has declared that the surface roughness of water hyacinth fiber decreses. The most significant result has been observed from the contact angle analysis and water absorption test, which is very clearly shows the increase in hydrophobicity of the plasma treated $\mathrm{ZnO}$ coated water hyacinth fiber.

\section{Acknowledgments}

The Author's profoundly thank the VIT University management, Chennai for giving the encouragement and lab support to carry out the research work.

\section{References}

[1] Anwer Md Masroor, A. H. Bhuiyan, IOSR Journal of Applied Physics (IOSRJAP) 16 (2012) 16-22.

[2] C. C. Gunnarsson, C. M. Petersen, Waste Management 27 (2007) 117-129.

[3] Ebhin R Masto, Sandeep Kumar, T. K. Rout, Pinaki Sarkar, Joshy George, L. C. Ram, Catena 111 (2013) 64-71.

[4] A. Malik, Environmental International 33 (2007) 122-138.

[5] Ying Gao, Xianhua Wang, Jun Wang, Xiangpeng Li, Jianjun Cheng, Haiping Yang, Hanping Chen, Energy 58 (2013) 376-383.

[6] P. Biller, A. B. Ross, Biofuels 3 (2012) 603-623.
[7] Y. K. Ong, S. Bhatia, Energy 35 (2010) 111-119.

[8] J. N. Nigam, J. of Biotechnology 07 (2002) 107-116.

[9] A. Kuniati, H. Darmokoesoemo, N. N. T. Puspaningsih, J. of Agriculture biotechnology and sustainable development 3 (2011) 182-188.

[10] D. Mashima, M. Kuniki, K. Sei, S. Soda, M. Ike, M. Fujita, Bioresource Technology 99 (2008) 2495-2500.

[11] Xia Ao, Jun Cheng, Richen Lin, Lingkan Ding, Junhu Zhou, Kefa Cen, Energy 55 (2013) 631-637.

[12] S. S. Toor, L. Rosendahl, A. Rudolf, Energy 36 (2011) 2328-2342.

[13] A, Kruse, J. of Supercritical fluids 47 (2009) 391-399.

[14] C. Douglas, John Elliott, L Sealock, R. Scott Butner, American chemical society 17 (1988) 179-188.

[15] R. Oraji, Master of Science Thesis, University of Saskatchewan, Saskatoon, 2008.

[16] Shishoo Roshan Shishoo. Plasma technologies for textiles. 1st ed. Woodhead Publishing in Textiles. England, Cambridge, 2007.

[17] Jing Zhang, Paul France, Arseni Radomyselskiy, Saswati Datta, Jiangang Zhao, William Van Ooij, Journal of Applied. Poly. Science 88 (2003) 1473-1481.

[18] Lei Fu, Zhimin Liu, Yunqi.Liu, Buxing Han, Pingan Hu, Lingchao Cao, Daoben Zhu, Advanced Materials 17 (2005) 217-221.

[19] Robert M. Dickson, L.Andrew Lyon, Journal of Physical Chem B 104 (2000) 6095-6098.

[20] Peter K. Stoimenov, Rosalyn L. Klinger, George L. Marchin, Kenneth J. Klabunde, Langmuir 18 (2002) 6679-6686.

[21] Xue-Guang Shao, Alexander Kai-Man Leung, Foo-Tim Chau, Accounts of Chemical Research 36 (2003) 276-283.

[22] G. Stephane, A, Mallat, IEEE Transactions on Pattern analysis and Machine Intelligence 11 (1989) 674-693

[23] Lin Yuan, Xu Rui Xiao, Li Xue Ping, Xiao Wen Zhou, Surface Science 579 (2005) 37-46.

[24] Alisher Maksumov, Ruxandra Vidu, Ahmet Palazoglu, Pieter Stroeve, Journal of colloid and interface science 272 (2004) 365377.

[25] A. Grossman and J. Morlet, SIAM J.Math 15 (1984) 723-730.

[26] N. Hari Prakash, Bornali Sarma, S. Gopi, Arun Sarma, Instrumentation Sci.and Tech. 44 (2016) 73-84.

[27] M. M. Morshed, Monjarul M. Alam, S. M. Daniels, Plasma Chemistry and Plasma Processing 32 (2012) 249-258.

[28] Gary G. Chinga, P. Johnsen, R. Dougherty, E Berli, Journal of Microscopy 227 (2007) 254-265.

[29] B. D. Cullity, Elements of X-ray diffraction. Addison-Wesley Publishing Company Inc., Reading, Massachusetts, 1956.

[30] L. Segal, J. Creely, Conrad CM, Textile Research Journal 29 (1959) 786-794.

[31] Yu-Qing Zhang, Wei-De Shen, Ru-Li Xiang, Lan-Jian Zhuge, Wei-Jian Gao, Wen-Bao Wang, Journal of Nanoparticle Research 9 (2007) 885-900.

[32] S. Mohanty, S. K. Nayak, S. K. Verma, S. S. Tripathy, Journal of Reinforced plastics and composites 23 (2004) 2047-2063.

[33] K. S. Venkatesh, S. R. Krishnamoorthi, N. S. Palani, V. Thirumal, S. P. Jose, F. M. Wang, R. Ilangovan, Indian Journal of Physics 89 (2015) 445-452.

[34] E. Sinha, S. K. Rout, Bull. Mat. Sci. 32 (2009) 65-76.

[35] Wafaa M. Raslan, Usama S. Rashed, Hanan El-Sayad, Azza A. ElHalwagy, Material Science and Applications 2 (2011) 1432-1442.

[36] N. David, Petr Klapetek, Central European Journal of Phys. 10 (2012) 181-188.

[37] S. Chandrasekaran, J. Check, S. Sundararajan, P. Shrotriya, Sensors and Actuators A 121 (2005) 121-130. 